\title{
The effect of emotional primes on attentional focus in high versus low depression
}

\author{
Sapir Miron ${ }^{1} \cdot$ Hadar Naftalovich ${ }^{1} \cdot$ Eyal Kalanthroff ${ }^{1}$ \\ Published online: 6 August 2019 \\ (C) The Psychonomic Society, Inc. 2019
}

\begin{abstract}
The effect of negative emotional stimuli on attentional focus is unclear. While a number of studies suggest that negative emotional stimuli improve attention, other studies show the opposite effect — namely, that negative emotional stimuli can impair attention and, specifically, attentional focus. It has been suggested that the detrimental effect of negative stimuli on attention is caused by attentional capture and difficulties in disengaging from the stimuli, an effect that is known to be stronger in depressed individuals. In the current study, we aimed to investigate the effect of negative primes on attentional focus as a function of levels of depression. Sixty-seven participants completed the attentional focus task, with either a neutral or a negative emotional prime preceding each trial. Results showed that attentional focus is improved in negative conditions, but that this effect is contingent upon levels of depression: While there is almost no effect of emotion on individuals with low levels of depression, there is a robust effect on individuals with high levels of depression. These results shed light on the process through which individuals with high levels of depression excessively focus on negative information, while simultaneously dismissing neutral information-a crucial part of the vicious cycle of negative mood and depression. Potential clinical implications are discussed.
\end{abstract}

Keywords Attentional focus · Illusory conjunctions $\cdot$ Depression $\cdot$ Emotion

Treisman and Gelade (1980) created a task that measures attentional focus through illusory conjunctions. Illusory conjunctions are combinations of features that are presented simultaneously in different spatial positions and, due to the manner of their presentation, are mistakenly mixed together (Botella, Barriopedro, \& Suero, 2001). For example, after being presented with a green $T$ and a red $R$, participants report the $R$ as being green (Botella, Suero, \& Durán, 2017). According to Treisman and Gelade, this phenomenon occurs when the focus of a person's attention on specific features is impeded by the conditions of the presentation. Indeed, the frequency of illusory conjunctions has been found to increase when attention is diverted (Fallon, Mattiesing, Dolfen, Manohar, \& Husain, 2018). Thus, illusory conjunctions provide an important scientific tool for measuring attentional focus in the lab. In the current study, we used the illusory

Sapir Miron

sapirmiron@gmail.com

1 Department of Psychology, The Hebrew University of Jerusalem, Room 27503, Mt. Scopus, 91905 Jerusalem, Israel conjunctions paradigm to investigate the effect of negative emotional primes on attentional focus in individuals with either high or low levels of depression.

Several studies have shown that emotions influence attention; however, these studies portray a complex picture. One line of studies suggests that negative emotional stimuli can speed up conflict processing and reduce the interference caused by incongruent stimuli, thereby enhancing attentional performance (Kanske \& Kotz, 2010, 2011a, 2011b; Zinchenko, Kanske, Obermeier, Schröger, \& Kotz, 2015; Zinchenko et al., 2018). One possible reason why emotional stimuli may enhance attentional performance is due to their high relevance for survival. For this reason, emotional stimuli are prioritized during the processing stream and can reduce the interference from irrelevant stimuli (Kanske, 2012). In addition, it has also been shown that negative emotional stimuli lead to the overall improvement of attention in tasks such as the visual search task (Olatunji, Ciesielski, Armstrong, \& Zald, 2011). On the other hand, there is also substantial evidence that negative emotional stimuli disrupt attentional performance (e.g., Kalanthroff, Aslan, \& Dar, 2017). The attentional control theory (Eysenck, Derakshan, Santos, \& Calvo, 2007) suggests that the involuntarily (bottom-up) allocation of 
resources to emotional distractors (Buodo, Sarlo, \& Palomba, 2002; Hartikainen, Ogawa, \& Knight, 2000) leads to the depletion of cognitive resources (Derakshan \& Esenck, 2009). For instance, Richards and Blanchette (2004) reported that naming the color of a word or a picture is slowed down when the word or picture is emotional compared with when the picture is neutral. Further, a few studies found that negative pictures have a detrimental effect on conflict resolution in tasks such as the Stroop task and the stop-signal task, even when the emotional stimulus is task irrelevant and precedes the target (Hart, Green, Casp \& Belger, 2010; Kalanthroff, Henik, Derakshan, \& Usher, 2016a; Padmala, Bauer, \& Pessoa, 2011; Sommer, Hajak, Döhnel, Meinhardt \& Müller, 2008; Verbruggen \& De Houwer, 2007). It is possible that task-irrelevant emotional stimuli impair attentional performance due to their tendency to capture attention (Brosch \& Van Bavel, 2012; Yiend, 2010).

One possible moderator for the effect of emotion on the attentional system is levels of depression (Disner, Beevers, Haigh, \& Beck, 2011). Specifically, it has been shown that the tendency of negative stimuli to capture attention is even more robust in depressed individuals (Donaldson, Lam, \& Mathews, 2007; Gotlib, Krasnoperova, Yue, \& Joormann, 2004) who also experience difficulties disengaging from those stimuli (Disner et al., 2011; Gotlib \& Joormann, 2010; Sanchez, Romero, \& De Raedt, 2017; Sanchez, Vazquez, Marker, LeMoult, \& Joormann, 2013). This leads to the prediction that higher levels of depression will be associated with larger impairments in attentional performance. On the other hand, depressed individuals are known to give higher priority to emotional stimuli (Beck, 1976; Bower, 1981; Joormann \& Quinn, 2014; Teasdale, 1988) and thus might recruit more attentional recourses when encountering negative stimuli (Donaldson et al., 2007). This leads to the prediction that negative stimuli will improve attentional performance in depressed individuals. Specifically, as attentional focus determines, to a high extent, our ability to accurately processes our environment, it is of great importance to understand the effect of emotional stimuli on attentional focus and particularly in depressed individuals. To date, this effect has not been investigated.

Based on the literature reviewed above, we expect to find an effect of negative emotional primes on attentional focus. However, whether negative emotional primes help or hinder attentional focus is yet unclear. The goal of the current study is to investigate whether negative emotional primes impair or improve attentional focus and whether levels of depression modulate this effect. To that aim, participants with various levels of depression completed the attentional focus task (Treisman, 1982; Treisman \& Schmidt, 1982), which measures attentional focus in the lab. Based on the evidence reviewed above, we investigate the connection between depression levels and attentional focus on an exploratory level.

\section{Method}

\section{Participants}

Seventy-three students of the Hebrew University of Jerusalem (Israel) participated in this experiment for a small monetary reward ( $\sim 5$ USD). All participants were naïve as to the purpose of the experiment. Six participants were excluded due to extremely low accuracy rates ( $<30 \%$ on both conditions). Thus, our final sample included 67 participants. Demographic and clinical characteristics of the samples are shown in Table 1.

A power analysis using G*Power 3.1 (Faul, Erdfelder, Lang, \& Buchner, 2007), indicated that the current sample size allows us to test for group differences in accuracy rates (with small-medium effect sizes) at a power $>95 \%$, and for $R^{2}$ change in the regression analysis at a power $>90 \%$, with a Type I error at which $\alpha<0.05$.

\section{Procedure}

The study was approved by the ethics committee of the Hebrew University of Jerusalem. Data collection and stimulus presentation were controlled by an HP Elite 800G3TWR computer with an Intel i7-8700 $4.20 \mathrm{GHz}$ processor. The stimuli were presented on an HP EliteDisplay E240 LED 23.8-in. monitor. After signing an informed consent form, participants completed two self-report questionnaires (i.e., Beck Depression Inventory-II [BDI-II], State-Trait Anxiety Inventory [STAI]). Given the known association between depression and anxiety, the STAI was used to control for anxiety levels in our analyses and to ensure anxiety levels do not mediate our findings. Next, participants completed the illusory conjunctions task, a measure of attentional focus (Treisman, 1982; Treisman \& Schmidt, 1982; see Fig. 1). Participants were tested individually and sat approximately 20 inches away from the computer screen.

\section{Measures}

Self-report questionnaires The Beck Depression Inventory-II (BDI-II; Beck, Steer, \& Brown, 1996) is a 21-item self-report measure that assesses the severity of depressive symptoms. The BDI-II has excellent test-retest reliability (.96; Sprinkle et al., 2002), excellent convergent validity and discriminant validity (Dozois, Dobson, \& Ahnberg, 1998), and has been shown to differentiate patient from control samples (Arnau, Meagher, Norris, \& Bramson, 2001). Internal consistency in the current sample was good (Cronbach's a $=.83$ ).

Attentional focus task The attentional focus task (see Fig. 1) included 10 practice trials (which included feedback for accuracy and RT; practice trials were not analyzed) and 144 
Table 1 Demographic and clinical characteristics of the high versus low depression groups.

\begin{tabular}{llll}
\hline & Low depression $(N=34)$ & High depression $(N=33)$ & Between-groups comparison \\
\hline Age (yrs.) & $23.74(2.46)[19-29]$ & $23.42(2.57)[18-28]$ & $t(65)=0.51, p=.62$ \\
Females/sample & $28 / 34$ & $26 / 33$ & $\chi^{2}(1)=0.14, p=.71$, Cramer's V $=.05$ \\
BDI-II & $3.76(2.13)[0-7]$ & $14.97(5.68)[8-28]$ & $t(65)=10.74, p<.001$ \\
STAI-T & $37.20(8.04)[22-54]$ & $43.63(7.62)[28-67]$ & $t(65)=3.35, p<.01$ \\
STAI-S & $38.14(6.38)[24-49]$ & $42.90(7.84)[24-61]$ & $t(65)=2.72, p<.01$ \\
\hline
\end{tabular}

Note. Mean, $(S D)$, [range], and between-groups comparisons of age, gender, State-Trait Anxiety Inventory (STAI), and BDI-II (Beck Depression Inventory-II) scores

experimental trials. Each trial started with a 1,000-ms fixation (a black point at the center of a white screen) followed by an emotional image. The images were randomly selected out of a set of 80 images, 40 negative (mean valence $=2.41$, mean arousal $=6.16$ ) and 40 neutral (mean valence $=5.01$, mean arousal $=2.84$ ) that were taken from the International Affective Picture System database (Lang, Bradley, \& Cuthbert, 2008; for a list of images, see Kalanthroff, Cohen, $\&$ Henik, 2013). The aversive pictures used in this study were selected to maximize the emotional impact. Images were presented in a random order for $100 \mathrm{~ms}$ followed by $50 \mathrm{~ms}$ of a blank screen. Next, the target display appeared and stayed in view for 2,000 ms. Each target consisted of three different central colored letters (randomly selected from $N, O, S, T$, or $X)$ arranged in a row in between two different colored digits (randomly selected from 1 to 9). The colors of the letters and digits were randomly selected from the following colors: red, orange, green, blue, black, gray, and brown, with the constraint that in each trial five different colors are used. The letters and emotional images appeared at the center of the screen on a white background. The letters were presented in Times New Roman font, type size 25. After the target display disappeared, participants were asked to report the sum of the two numbers that appeared in the current trial (Question 1). Next, participants were asked to decide whether a specific stimulus, a combination of one letter and a color, appeared in the current trial (Question 2: integrated features; e.g., "Did the letter $S$ appear in RED?"). For each question, participants were asked to respond using the keyboard, as accurately and as quickly as possible, and were limited to $3,000 \mathrm{~ms}$.

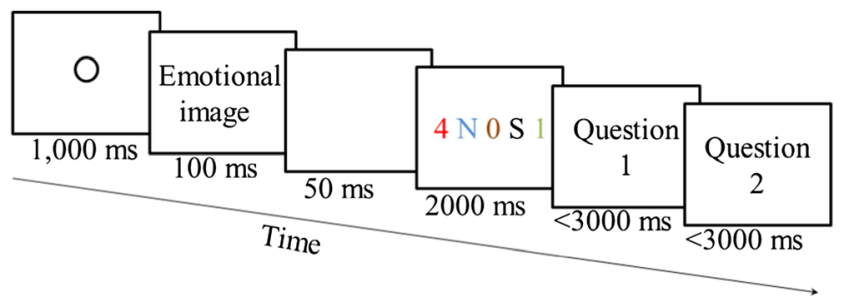

Fig. 1 Example of a trial in the attentional focus task. Note. Question $1=$ Report the sum of the two numbers. Question 2 = Did the letter (e.g., $S$ ) appear in X (e.g., red) color? In the last two slides, responses were limited to $3,000 \mathrm{~ms}$

\section{Results}

Accuracy rates for the illusory conjunctions question (Question 2) were calculated for each participant in each valence condition (negative vs. neutral). First, a significant correlation was found between the BDI-II scores and the emotional interference effect (negative-neutral) on the illusory conjunctions question $(r=.33, p<.01$; see Fig. 2$)$. Next, we conducted a block regression model to predict BDI-II scores using accuracy rates on the neutral and negative valence conditions after controlling for age and STAI-trait scores (to control for demographic and clinical measures). The final model revealed that age did not contribute to the prediction of BDI-II scores (standardized $\beta=$ $-.15, p=.18$ ), while STAI-trait scores did (standardized $\beta$ $=.43, p<.01)$. Most importantly, after accounting for age and STAI-trait scores, accuracy rates on negative trials positively contributed to the prediction of BDI-II scores (standardized $\beta=.37, p<.05$ ), and accuracy rates on neutral trials negatively contributed to the prediction of BDI-II scores (standardized $\beta=-.53, p<.01$ ). These results indicate that higher BDI-II scores are associated with better performance on negative trials while lower BDI-II scores are associated with better performance on neutral trials (see Figs. 2-3). Importantly, STAI-trait scores did not correlate with the emotional interference effect (negative-neutral) on the illusory conjunctions question $(r=.10, p=.41)$.

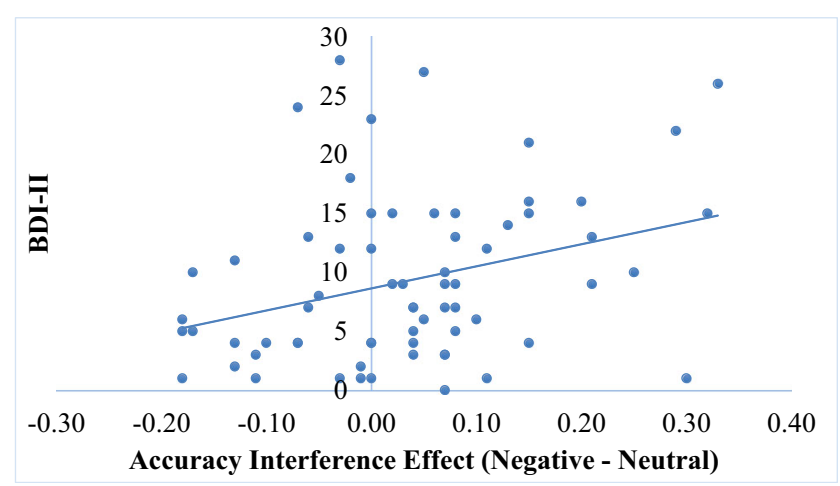

Fig. 2 Correlation between accuracy interference effect and BDI-II score. The correlation is significant at the .01 level 


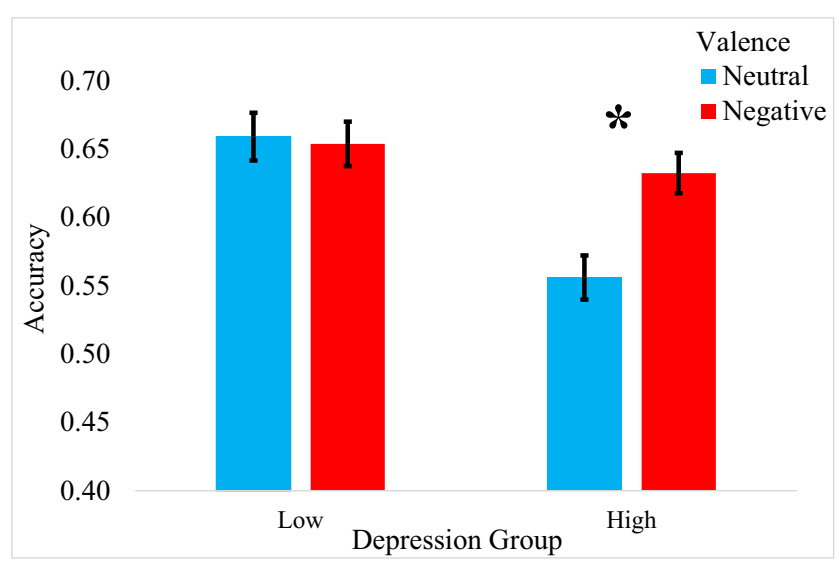

Fig. 3 Accuracy rates in the different valence conditions of the attentional focus task (Question 2) for the high and low depression group. Error bars represent one standard error of the mean. $* p<.05$

In order to examine our a priori assumptions, we divided all subjects into two equal groups based on the median-those with BDI-II scores below $7(N=34)$ were in the "low" depression group; those in the high depression levels group ranged from subclinical $(<13)$ to moderately depressed (between 20 and 27), and for the purposes of this analysis will be placed in the "high" depression group $(N=33)$. As can be seen in Table 1, there were no significant differences between the high and low depression groups in all demographic measures. Accuracy rates for the illusory conjunctions question were subjected to a mixed-model analysis of variance (ANOVA), with valence (neutral vs. negative) as a within-subjects factor and group (high vs. low depression) as a between-subjects factor. There was no main effect for group, $F(1,65)=2.04, p=.16$, a significant main effect for valence, $F(1,65)=6.20, p=.02$, and a significant Valence $\times$ Group interaction, $F(1,65)=8.22, p<$ $.01, \eta_{\mathrm{p}}{ }^{2}=.11$ (see Fig. 3). In order to further investigate this interaction according to our a priori predictions, we carried out two planned comparisons, with Bonferroni correction for multiple comparisons, using paired-sample $t$ tests, with valence as a within-subjects factor, for the low and high depression groups separately. In the low depression group, there was no significant difference between the two valence conditions, $t(33)=0.29, p=$ .77 , while in the high depression group we found significantly higher accuracy rates in the negative valence condition compared with the neutral valence condition, $t(32)=3.52, p<.01$, Cohen's $d=0.42$ (see Fig. 3). This indicates improved attentional focus in the high depression group following exposure to negative priming. Notably, independent-sample $t$ tests comparing the high versus low depression groups within each valence condition revealed no significant between-group differences for the negative valence condition, $t(65)=0.50, p=.62$, and a significant effect for the neutral valence condition, $t(65)=$ $2.15, p=.04$, Cohen's $d=0.53$, indicating that for the neutral valence condition accuracy rates were higher in the low depression group.

\section{Discussion}

The goal of the current study was to examine whether levels of depression modulate the effect of negative emotional primes on attentional focus, to determine whether emotional stimuli hinder or improve attentional focus. To that end, we administered the attentional focus task using either negative or neutral task-irrelevant emotional primes. A significant correlation between the emotional interference effect (accuracy for negative trials-accuracy for neutral trials) and BDI-II scores was found, so that a larger emotional interference effect was found in participants with higher BDI-II scores. In other words, participants with higher levels of depression displayed fewer illusory conjunctions (resulting in better performance) on negative, as compared with neutral, trials, while participants with lower levels of depression displayed similar illusory conjunctions on neutral and negative trials. This indicates that higher levels of depression are advantageous for negative and disadvantageous for neutral primes. This correlation stayed significant after age and anxiety levels were controlled for using a block regression model. Splitting the sample into high and low depression groups revealed that the participants in the high depression group exhibited higher accuracy rates when the prime was negative compared with when the prime was neutral, while participants in the low depression group displayed no differences in accuracy rates between the two valence conditions. In addition, we found that participants with low levels of depression had higher accuracy rates in the neutral valence condition than did individuals with high depression, while there were no significant between-group differences for the negative valence condition.

The current study findings support the suggestion that negative emotional primes improve, rather than disrupt attentional performance (Olatunji, Ciesielski, Armstrong, \& Zald, 2011), at least in respect to attentional focus. However, in individuals with low depression, we found no behavioral indication for the effect of emotion on attention. It is possible that the effect of negative emotional primes on attention can be downregulated in individuals with low levels of depression (e.g., Kalanthroff, Henik, Derakshan, \& Usher, 2016a), and thus no behavioral indication exists. This finding might shed light on the cognitive mechanism that supports and maintains the cycle of negative mood and perhaps of depression. Given that in real life negative cues commonly appear in negative contexts, increased attentional focus when encountering a negative cue is most likely to lead to increased processing of negative information (Disner et al., 2011). At the same time, less attentional focus is allocated to neutral situations, and thus less neutral information is being processed. In turn, focused attention on negative information and less focused attention in neutral situations can play a role in the maintenance of negative mood (Armstrong \& Olatunji, 2012), which might then lead to increased attentional focus in negative situations, and 
so on. Moreover, our findings support the notion that depressed individuals see reality in a more accurate fashion (Kornbrot, Msetfi, \& Grimwood, 2013), but also suggest that such accurate processing in depressed individuals occurs mainly in negative situations. Given that our sample was not composed of clinically depressed individuals, conclusions regarding clinical depression should be considered with caution.

The current study findings also suggest that in the neutral valence condition, participants in the high depression group had more illusory conjunctions, indicating lower attentional focus, compared with the low depression group. This finding is consistent with previous studies indicating reduced attention and impaired attentional focus in depressed individuals (Hasher \& Zacks, 1979; Murphy et al., 1999).

The current results might have specific clinical implications. First, our findings can be used by clinicians as part of psychoeducation. Clinicians can explain the negative attention bias to patients presenting with depressive symptoms, providing a rationale for why certain individuals might feel that "everything is negative." Second, understanding that negative primes might improve attentional focus might be used as a tool to increase attentional focus in treatment. Thus, if a condition is artificially created, in which negative images prime positive information, patients with high levels of depression might be able to process more positive information.

A few limitations of the current study need to be considered. Analog samples have significant value, which in the spirit of Research Domain Criteria (Insel, 2014) provide an opportunity to understand the etiology, development, and maintenance of the full range ("from normal to abnormal") of different disorders (Abramowitz et al., 2014). Nevertheless, as mentioned above, our sample was not composed of participants diagnosed with clinical depression, and thus all conclusions regarding depression should be considered with caution. Second, the current study is based on the seminal work of Anne Treisman, who, through multiple studies, showed that illusory conjunctions are a measure of attentional focus (Treisman, 1982). However, this task involves several processes that may be influenced by the emotional primes. Thus, future studies need to examine the specific processes in order to achieve a better understanding of the mechanisms investigated here. Finally, the current study consisted of a low number of male participants.

In conclusion, in the current study, we used the attentional focus task to investigate attentional focus as a function of levels of depression. Our findings suggest that attentional focus is improved in negative conditions, but that this effect is contingent upon levels of depression-while there is almost no effect of emotion on individuals with low levels of depression, there is a robust effect on individuals with high levels of depression. These results might shed light on the underlying mechanism of the vicious cycle of negative mood and depression, which is characterized by excessive processing of negative information (Kalanthroff, Linkovski, Henik, Wheaton, \& Anholt, 2016b) while neglecting to process neutral (nonnegative) information.

Open practices statement The data and task for the experiments are available on https://docs.wixstatic.com/ugd/ 1e6ddc_7c687f9ffac0422999059c73c6c9efcc.xlsx?dn= spss $\% 20$ participants $\% 20$ demographic\%20and\%20clinic. The experiment was not preregistered.

\section{References}

Abramowitz, J. S., Fabricant, L. E., Taylor, S., Deacon, B. J., McKay, D., $\&$ Storch, E. A. (2014). The relevance of analogue studies for understanding obsessions and compulsions. Clinical Psychology Review, 34, 206-217.

Armstrong, T., \& Olatunji, B. O. (2012). Eye tracking of attention in the affective disorders: A meta-analytic review and synthesis. Clinical Psychology Review, 32, 704-723.

Arnau, R. C., Meagher, M. W., Norris, M. P., \& Bramson, R. (2001). Psychometric evaluation of the Beck Depression Inventory-II with primary care medical patients. Health Psychology, 20, 112-119.

Beck, A. T. (1976). Cognitive therapy and the emotional disorders. New York, NY: International Universities Press. Beck, A. T., Steer, R. A., \& Brown, G. K. (1996). Beck depression inventory manual (2nd ed.). San Antonio, TX: Psychological Corporation.

Botella, J., Barriopedro, M., \& Suero, M. (2001). A model of the formation of illusory conjunctions in the time domain. Journal of Experimental Psychology: Human Perception and Performance, 27, 1452-1467.

Botella, J., Suero, M., \& Durán, J. I. (2017). On the reality of illusory conjunctions. The Journal of General Psychology, 144, 187-205.

Bower, G. H. (1981). Mood and memory. American Psychologist, 36, 129-148.

Brosch, T., \& Van Bavel, J. J. (2012). The flexibility of emotional attention: Accessible social identities guide rapid attentional orienting. Cognition, 125, 309-316.

Buodo, G., Sarlo, M., \& Palomba, D. (2002). Attentional resources measured by reaction times highlight differences within pleasant and unpleasant, high arousing stimuli. Motivation and Emotion, 26, 123-138.

Derakshan, N., \& Eysenck, M. W. (2009). Anxiety, processing efficiency, and cognitive performance: New developments from attentional control theory. European Psychologist, 14, 168-176.

Disner, S. G., Beevers, C. G., Haigh, E. A., \& Beck, A. T. (2011). Neural mechanisms of the cognitive model of depression. Nature Reviews Neuroscience, 12, 467-477.

Donaldson, C., Lam, D., \& Mathews, A. (2007). Rumination and attention in major depression. Behaviour Research and Therapy, 45, 2664-2678.

Dozois, D. J. A., Dobson, K. S., \& Ahnberg, J. L. (1998). A psychometric evaluation of the Beck Depression Inventory-II. Psychological Assessment, 10, 83-89.

Eysenck, M. W., Derakshan, N., Santos, R., \& Calvo, M. G. (2007). Anxiety and cognitive performance: attentional control theory. Emotion, 7, 336-353.

Fallon, S. J., Mattiesing, R. M., Dolfen, N., Manohar, S. G., \& Husain, M. (2018). Ignoring versus updating in working memory reveal differential roles of attention and feature binding. Cortex, 107, 50-63. 
Faul, F., Erdfelder, E., Lang, A. G., \& Buchner, A. (2007). G*Power 3: A flexible statistical power analysis program for the social, behavioral, and biomedical sciences. Behavior Research Methods, 39, 175-191.

Gotlib, I. H., \& Joormann, J. (2010). Cognition and depression: current status and future directions. Annual Review of Clinical Psychology, $6,285-312$

Gotlib, I. H., Krasnoperova, E., Yue, D. N., \& Joormann, J. (2004). Attentional biases for negative interpersonal stimuli in clinical depression. Journal of Abnormal Psychology, 113, 127-135.

Hart, S. J., Green, S. R., Casp, M., \& Belger, A. (2010). Emotional priming effects during Stroop task performance. NeuroImage, 49(3), 2662-2670.

Hartikainen, K. M., Ogawa, K. H., \& Knight, R. T. (2000). Transient interference of right hemispheric function due to automatic emotional processing. Neuropsychologia, 38, 1576-1580.

Hasher, L., \& Zacks, R. T. (1979). Automatic and effortful processes in memory. Journal of Experimental Psychology: General, 108(3), $356-388$.

Insel, T. R. (2014). The NIMH Research Domain Criteria (RDoC) Project: Precision medicine for psychiatry. American Journal of Psychiatry, 171, 395-397.

Joormann, J., \& Quinn, M. E. (2014). Cognitive processes and emotion regulation in depression. Depression and Anxiety, 31, 308-315.

Kalanthroff, E., Aslan, C., \& Dar, R. (2017). Washing away your sins will set your mind free: Physical cleansing modulates the effect of threatened morality on executive control. Cognition and Emotion, 31, 185-192.

Kalanthroff, E., Cohen, N., \& Henik, A. (2013). Stop feeling: inhibition of emotional interference following stop-signal trials. Frontiers in Human Neuroscience, 7, 1-7.

Kalanthroff, E., Henik, A., Derakshan, N., \& Usher, M. (2016a). Anxiety, emotional distraction, and attentional control in the Stroop task. Emotion, 16, 293-300.

Kalanthroff, E., Linkovski, O., Henik, A., Wheaton, M. G., \& Anholt, G. E. (2016b). Inhibiting uncertainty: Priming inhibition promotes reduction of uncertainty. Neuropsychologia, 92, 142-146.

Kanske, P. (2012). On the influence of emotion on conflict processing. Frontiers in Integrative Neuroscience, 6(42), 1-4.

Kanske, P., \& Kotz, S. A. (2010). Modulation of early conflict processing: N200 responses to emotional words in a flanker task. Neuropsychologia, 48(12), 3661-3664.

Kanske, P., \& Kotz, S. A. (2011a). Emotion speeds up conflict resolution: A new role for the ventral anterior cingulate cortex? Cerebral Cortex, 21(4), 911-919.

Kanske, P., \& Kotz, S. A. (2011b). Emotion triggers executive attention: Anterior cingulate cortex and amygdala responses to emotional words in a conflict task. Human Brain Mapping, 32(2), 198-208.

Kornbrot, D. E., Msetfi, R. M., \& Grimwood, M. J. (2013). Time perception and depressive realism: Judgment type, psychophysical functions and bias. PLOS One, $8,1-9$.

Lang, P., Bradley, M., \& Cuthbert, B. (2008). International affective manual (Tech. Rep. No. A-8). Gainesville: University of Florida

Murphy, F. C., Sahakian, B. J., Rubinsztein, J. S., Michael, A., Rogers, R. D., Robbins, T. W., . . . Paykel, E. S. (1999). Emotional bias and inhibitory control processes in mania and depression. Psychological Medicine, 29, 1307-1321.

Olatunji, B. O., Ciesielski, B. G., Armstrong, T., \& Zald, D. H. (2011). Emotional expressions and visual search efficiency: Specificity and effects of anxiety symptoms. Emotion, 11, 1073-1079.

Padmala, S., Bauer, A., \& Pessoa, L. (2011). Negative emotion impairs conflict-driven executive control. Frontiers in Psychology, 2, 192.

Richards, A., \& Blanchette, I. (2004). Independent manipulation of emotion in an emotional Stroop task using classical conditioning. Emotion, 4, 275-281.

Sanchez, A., Romero, N., \& De Raedt, R. (2017). Depression-related difficulties disengaging from negative faces are associated with sustained attention to negative feedback during social evaluation and predict stress recovery. PLOS ONE, 12, 1-24.

Sanchez, A., Vazquez, C., Marker, C., LeMoult, J., \& Joormann, J. (2013). Attentional disengagement predicts stress recovery in depression: An eye-tracking study. Journal of Abnormal Psychology, 122, 303-313.

Sommer, M., Hajak, G., Döhnel, K., Meinhardt, J., \& Müller, J. L. (2008). Emotion-dependent modulation of interference processes: An fMRI study. Acta Neurobiologiae Experimentalis, 68(2), 193-203.

Sprinkle, S. D., Lurie, D., Insko, S. L., Atkinson, G., Jones, G. L., Logan, A. R., . . . Bissada, N. N. (2002). Criterion validity, severity cut scores, and test-retest reliability of the Beck Depression Inventory-II in a university counseling center sample. Journal of Counseling Psychology, 49, 381-385.

Teasdale, J. D. (1988). Cognitive vulnerability to persistent depression. Cognition \& Emotion, 2, 247-274.

Treisman, A. (1982). Perceptual grouping and attention in visual search for features and for objects. Journal of Experimental Psychology: Human Perception and Performance, 8, 194-214.

Treisman, A. M., \& Gelade, G. (1980). A feature-integration theory of attention. Cognitive Psychology, 12, 97-136.

Treisman, A., \& Schmidt, H. (1982). Illusory conjunctions in the perception of objects. Cognitive Psychology, 14, 107-141.

Verbruggen, F., \& De Houwer, J. (2007). Do emotional stimuli interfere with response inhibition? Evidence from the stop signal paradigm. Cognition and Emotion, 21(2), 391-403.

Yiend, J. (2010). The effects of emotion on attention: A review of attentional processing of emotional information. Cognition and Emotion, 24, 3-47.

Zinchenko, A., Kanske, P., Obermeier, C., Schröger, E., \& Kotz, S. A. (2015). Emotion and goal-directed behavior: ERP evidence on cognitive and emotional conflict. Social Cognitive and Affective Neuroscience, 10(11), 1577-1587.

Zinchenko, A., Kanske, P., Obermeier, C., Schröger, E., Villringer, A., \& Kotz, S. A. (2018). Modulation of cognitive and emotional control in age-related mild-to-moderate hearing loss. Frontiers in Neurology, 9, 1-16.

Publisher's note Springer Nature remains neutral with regard to jurisdictional claims in published maps and institutional affiliations. 